\title{
Hard-core Yukawa model for two-dimensional charge-stabilized colloids
}

\author{
R. Asgari, ${ }^{1}$ B. Davoudi, ${ }^{1,2}$ and B. Tanatar ${ }^{3}$ \\ ${ }^{1}$ (IPM) Institute for Studies in Theoretical Physics and Mathematics, P.O. Box 19395-5531, Tehran, Iran \\ ${ }^{2}$ Scuola Normale Superiore, Piazza dei Cavalieri 7, 56126 Pisa, Italy \\ ${ }^{3}$ Department of Physics, Bilkent University, Bilkent, Ankara, Turkey
}

(Received 18 April 2001; revised manuscript received 29 May 2001; published 25 September 2001)

\begin{abstract}
The hypernetted chain and Percus-Yevick approximations are used to study the phase diagram of a simple hard-core Yukawa model of charge-stabilized colloidal particles in a two-dimensional system. We calculate the static structure factor and the pair distribution function over a wide range of parameters. Using static correlation functions, we present an estimate for the liquid-solid phase diagram for a wide range of parameters.
\end{abstract}

DOI: 10.1103/PhysRevE.64.041406

PACS number(s): 82.70.Dd, 64.70.Dv

\section{INTRODUCTION}

Systems composed of small particles with sizes of tens of nanometers dispersed in solvents are known as colloidal suspensions [1]. With increasing interest in the properties of complex fluids and their various thermodynamic phases, colloidal systems have been the subject of vast experimental and theoretical investigations $[2,3]$.

Two-dimensional (2D) colloids are of special interest for several reasons. First, colloidal systems can be realized rather easily experimentally, as in air-water interfaces or between two parallel glass plates, and advances in measurement techniques such as digital video microscopy brought about a wealth of information on these systems [4-8]. Second, the nature of the melting of 2D crystals is different than their 3D counterparts, making them a subject of intense theoretical and experimental investigation [9-11]. Colloidal suspensions in the fluid phase may crystallize as their density increases or the range of interaction changes. The existence of a glassy phase [12] and re-entrant melting [13,14] are examples of various motivating derives behind some of the recent work. The possibilities of photonic band-gap structures made out of colloidal crystals promise interesting applications [15].

In this work, we first use the hypernetted-chain (HNC) and Percus-Yevick (PY) approximations for a comparison between the two approaches for the pure hard-core potential (PHCP) as a model for a colloidal system. It is well known that the simple HNC approximation, which omits elementary diagrams, gives a good description of the large interparticle distance behavior. On the other hand, the PY approximation yields better results for short interparticle distances [16-18]. In the next step, we employ the widely used hypernettedchain approximation to study liquid state correlation functions and freezing transition of charge-stabilized colloidal particles in two dimensions interacting via the hard-core Yukawa potential (HCYP). Our basic aim is to test how well the HNC method models the static properties of a colloidal system. Similar calculations [19] in three dimensions resulted in a good agreement between the predictions of HNC theory and Monte Carlo simulations. To this end, we calculate the pair-correlation function and the static structure factor for a range of densities and temperatures. We discuss our results in comparison to other theoretical approaches and available experiments.

\section{MODEL AND THEORY}

\section{A. Hard-core Yukawa model}

In this section, we introduce a model of two-dimensional charged colloidal particles. We consider colloidal particles trapped in a surface energy well at an air-water interface modeled by a hard-core interaction and a Yukawa interaction [4]. The pair potential $V(r)$ between the colloidal particles is given by

$$
V(r)= \begin{cases}\infty & \text { for } r<\sigma \\ \lambda \frac{\exp [-\kappa(r-\sigma)]}{r} & \text { for } r>\sigma,\end{cases}
$$

where $\lambda$ is the strength of the interaction, $\sigma$ is the hard-core radius, and $\kappa$ is the inverse of the Debye screening length of the colloidal system. The PHCP without the Yukawa tail is obtained by setting $\lambda=0$. The form of the interaction potential between charge-stabilized colloids is different from that between charged colloidal particles, as inferred from the recent experiments of Marcus and Rice [7,8]. The role of longrange forces in colloidal systems was been emphasized by Noro et al. [20].

\section{B. HNC and PY formalisms}

Given the above potential to describe interactions between charged colloidal particles, the evaluation of correlation functions reduces to a problem in classical liquid state theory [21]. One of the basic quantities of interest is the pair-distribution function, defined by

$$
g\left(\left|\mathbf{r}-\mathbf{r}^{\prime}\right|\right)=\left\langle\sum_{\mathrm{i}=1}^{\mathrm{N}} \sum_{\mathrm{j} \neq \mathrm{i}}^{\mathrm{N}} \delta\left(\mathbf{r}-\mathbf{r}_{\mathrm{i}}\right) \delta\left(\mathbf{r}^{\prime}-\mathbf{r}_{\mathrm{j}}\right)\right\rangle / \rho^{2}
$$

where $\rho$ is the density of the liquid. The pair-distribution function gives the probability of finding a particle at $r^{\prime}$ when we fix another particle at $r$. Other relevant quantities are the pair-correlation function $h(r)=g(r)-1$, and the static structure factor defined by 


$$
S(q)=1+\rho \int d \mathbf{r} h(r) \exp (i \mathbf{q} \cdot \mathbf{r})
$$

The pair-correlation function for a simple, isotropic fluid can be decomposed using the Ornstein-Zernike relation $[21,22]$

$$
h(r)=C(r)+\rho \int d r^{\prime} C\left(\left|\mathbf{r}-\mathbf{r}^{\prime}\right|\right) \mathbf{h}\left(\mathbf{r}^{\prime}\right),
$$

where $C(r)$ is called the direct correlation function, and can be related to the structure factor through

$$
S(q)=1 /[1-\rho C(q)] .
$$

A closure relation between $h(r)$ and $C(r)$ is needed to supplement the Ornstein-Zernike relation. There are some closure relations between $h(r)$ and $C(r)$. In the following we use the well-known HNC and PY approximations. In the HNC approximation, the closure relation takes the form $[21,22]$

$$
C(r)=\exp [-\beta V(r)+Y(r)]-1-Y(r),
$$

where $V(r)$ is the interparticle pair potential, and by construction $Y(r)=h(r)-C(r) . \beta=1 /\left(k_{B} T\right)$ is the inverse temperature. On the other hand, the PY closure relation is given by [21]

$$
C(r)=\left(e^{-\beta V(r)}-1\right)[Y(r)+1] .
$$

A self-consistent solution of Eq. (4), (6), or (7) gives information about the correlations in the liquid state within the HNC or PY schemes.

\section{NUMERICAL RESULTS AND DISCUSSION}

In this section, we present the results of our numerical calculation of HNC and PY equations for a system of colloidal particles interacting with HCYP. We have solved Eqs. (4), (6) or Eq. (7) for HNC and PY approximations, respectively. One can find solutions for this set of equations by numerical iteration, taking the potential defined in Eq. (1) as input. We have obtained correlation functions for a wide range of values of the density $\rho^{*}=\sigma^{2} \rho$, screening length $\kappa \sigma$, and inverse temperature $\beta \lambda$. More specifically, we have calculated the pair-correlation function, the static structure factor, and a direct correlation function which is related to the effective potential. We have then used the Hansen-Verlet [23] criterion to estimate the phase line for liquid-solid transition.

As a comparison, first, we compare the results of the HNC and PY approximations. To show the differences, we compare the results for two extreme regions: low and high densities. In Fig. 1, we show the pair-distribution function at $\rho^{*}=0.4$ and 0.8 within the HNC and PY approximations for a PHCP. One can see that the differences between the two approaches increase with increasing density. It is evident that we have a sharp peak in $r=\sigma$, and the height of the peak increases as we increase the density. This means that the probability of finding a particle at $r=\sigma$ when we fix a par-

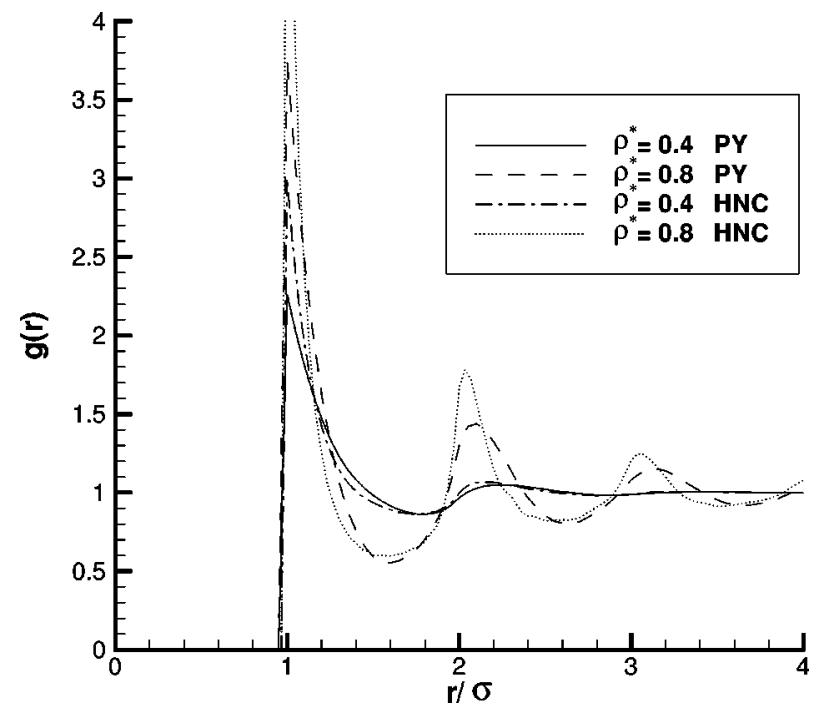

FIG. 1. The pair-distribution function for PHCP in terms of $r \sigma$ at $\rho=0.4$ and 0.8 within the HNC and PY approximations.

ticle in the origin increases with increasing density. One can observe a long-range oscillatory behavior in the pairdistribution function, which is a sign of a liquid-solid phase transition.

Using Eq. (3), one can find the static structure factor. In Fig. 2 we depict the static structure factor at $\rho^{*}=0.4$ and 0.8 within the HNC and PY approximations for a PHCP. There is a peak around $k=2 \pi / \sigma$ which increases with increasing density. A sharp peak in the structure factor at $k=2 \pi / \sigma$ produces a long-range oscillatory behavior with a periodicity $\sigma$ in the pair-distribution function, which is a signature of long-range order or of the solid phase. Furthermore, the height of this peak is very important in predicting the liquidsolid phase transition. The Hansen-Verlet criterion simply states that the phase transition takes place when the value of the first peak in $S(k)$ reaches a special constant. It is interesting that the difference between the values of the static structure factors within the two approximations near the first

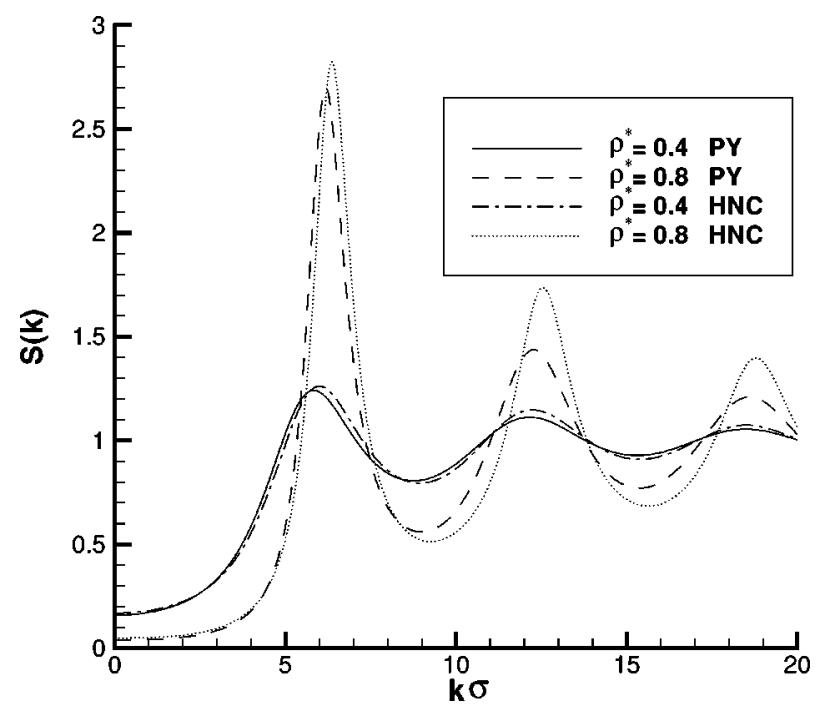

FIG. 2. The static structure factor for PHCP in terms of $k \sigma$ at $\rho=0.4$ and 0.8 within the HNC and PY approximations. 


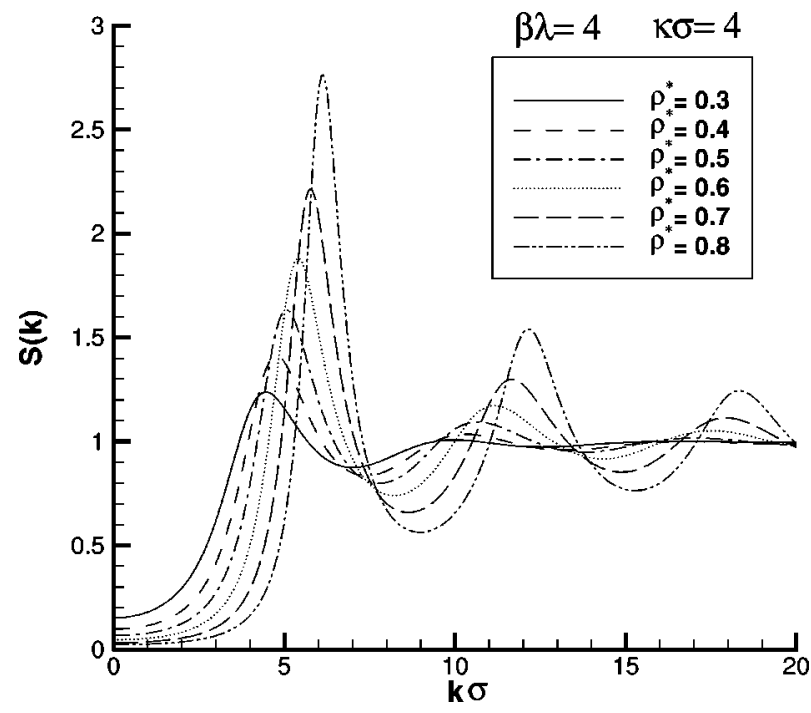

FIG. 3. The static structure factor in terms of $k \sigma$ at $\beta \lambda=4$, $\kappa \sigma=4$, and different values of $\rho$.

peak is small. This small difference allows the use of HNC results to be reasonable to predict the liquid-solid phase transition for a PHCP. We used the recent results of Weber, Marx, and Binder [9], for the density at which the phase transition takes place, to determine the height of this peak in our results. This value in our results is about $S_{\max } \cong 3.5$, corresponding to $\rho^{*}=0.89$ within the HNC approximation.

We now report the results for the HCYP within the HNC approximation. The static structure factor is shown in Fig. 3 at fixed $\beta \lambda=4$ and $\kappa \sigma=4$ for different values of $\rho^{*}$. We observe that with increasing density the peak structure in the static structure factor increases. Again, the increasing peak height of the static structure factor shows a tendency toward the formation of a solid phase. We also can observe that there is a shift in the position of the peak, which means in the high density regime the hard-core part of the potential is dominant compared to the Yukawa part, and the position of the peak shifts to the position for the hard-core one.

We observe the same behavior by fixing $\kappa \sigma$ and $\rho^{*}$ and changing $\beta \lambda$, or by fixing $\beta \lambda$ and $\rho^{*}$ and changing $\kappa \sigma$. The above argument shows that, by changing the strength and range of the Yukawa part or the temperature, one can reach the phase transition point. The results at $\rho^{*}=0.3$ and $\beta \lambda$ $=80$ for different values of $\kappa \sigma$, and at $\rho^{*}=0.4$ and $\beta \lambda=4$ for different values $\kappa \sigma$, are depicted in Figs. 4 and 5 .

It is evident from Fig. 4 that the position of the peak is different from the high density limit, which implies, for low density limit, that the Yukawa part makes the major contribution to the liquid-solid phase transition. The position of the peak also supplies some information about periodicity in the pair-distribution function. Generally, the position of the peak near the phase transition point for the HCYP only depends on the value of the density, and is insensitive to other parameters.

In Fig. 6 we show the pair-distribution function at fixed $\beta \lambda=4$ and $\kappa \sigma=4$, and different values of $\rho^{*}$. It is interesting to note that we have a shift toward $r=\sigma$ as we increase the density, which again means that the hard-core part makes

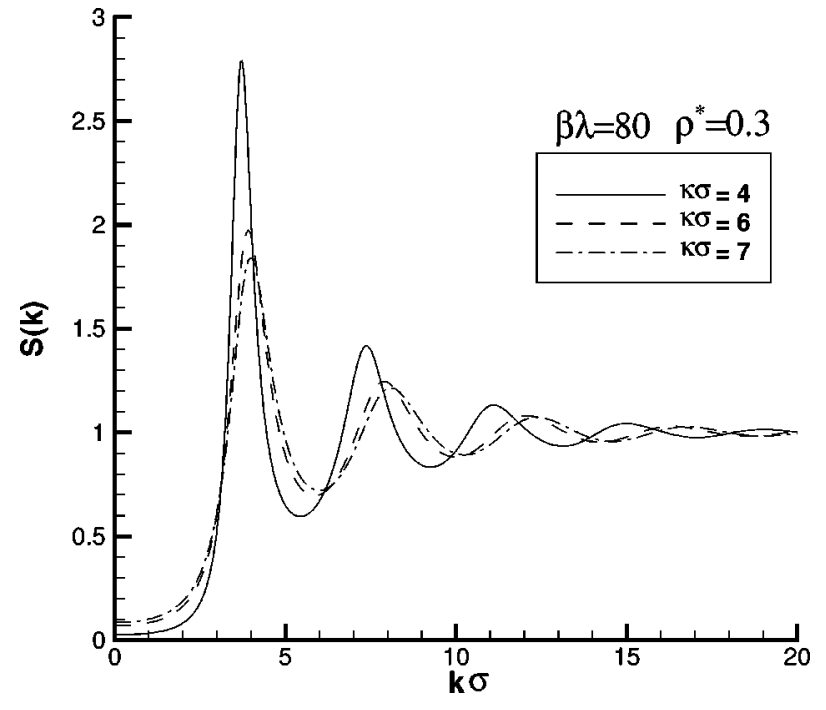

FIG. 4. The static structure factor in terms of $k \sigma$ at $\rho^{*}=0.3$, $\beta \lambda=80$, and different values of $\kappa \sigma$.

the major contribution to the high density limit. It is clear that the period of the oscillations also changes as the density increases.

It is possible to study the evolution of the first peak in the static structure factor when the physical quantities change, to extract some useful information about the liquid-solid phase transition. In Fig. 7, we show the variation of this peak in terms of $\beta \lambda$ for a set of densities $\rho^{*}=0.3,0.4,0.5,0.6$, and 0.7 at $\kappa \sigma=4$. It is clear from the figure that the Yukawa part is important only for low density, and that it is the source of the liquid-solid phase transition in the low density region. It is interesting that $\beta \lambda$, at which the phase transition takes place, also shifts to the right very rapidly with decreasing density, because the mean distance between the two particles increases with reducing density, and the effect of the Yukawa interaction decreases exponentially as the mean distance increases.

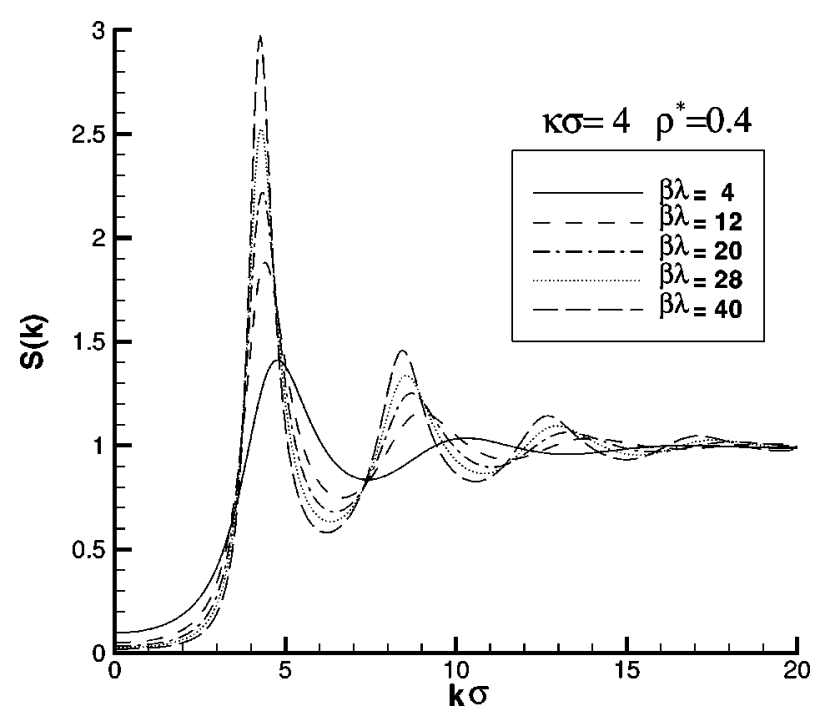

FIG. 5. The static structure factor in terms of $k \sigma$ at $\rho^{*}=0.4$, $\kappa \sigma=4$, and different values of $\beta \lambda$. 


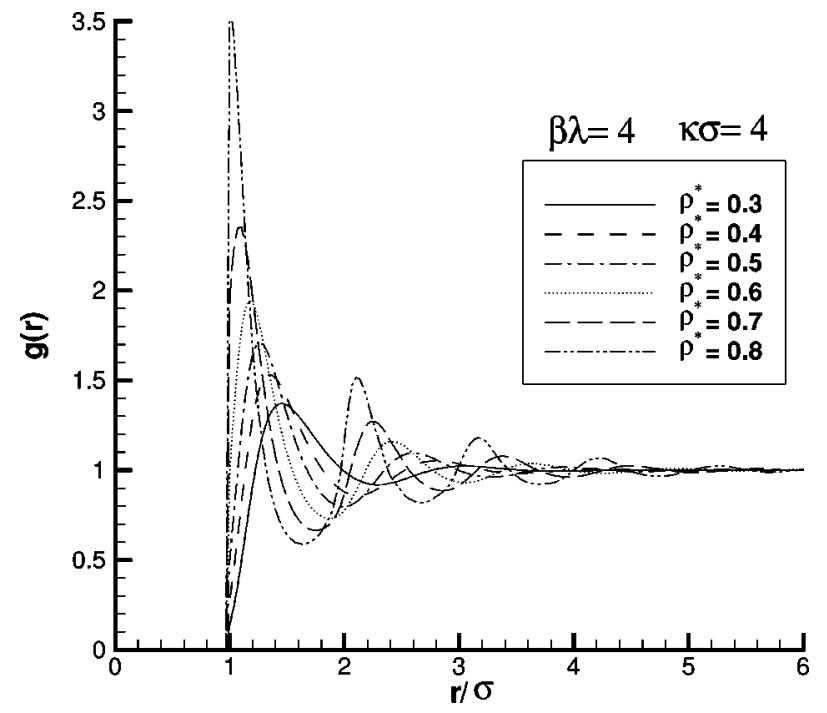

FIG. 6. The pair distribution function in terms of $r / \sigma$ at $\beta \lambda=4$ and $\kappa \sigma=4$ and different value of $\rho^{*}$.

In Fig. 8 we show the variation of the first peak in terms of $\beta \lambda$ at $\rho=0.4$ and $\kappa \sigma=4,6$, and 7. It is observed that with increasing $\kappa \sigma$, the curves $S_{\text {max }}$ tend toward a constant, which means that hard-core part again has the major effect on this system in this region; we can thus observe the phase transition with changing density.

From the previous figures and using the Hansen-Verlet criterion, we can obtain the liquid-solid phase transition line. In Fig. 9 we depict the phase line for different values of $\kappa \sigma$. It is interesting to note that all phase separation lines tend toward the same point in the high density limit, which is reasonable. Another important point is that with increasing $\kappa \sigma$ the phase line tends toward the line $\rho^{*}=0.89$. This line is equivalent to the liquid-solid phase transition line for a pure hard-core potential.

The phase diagram of colloidal systems in two dimensions is of particular interest, because of the subtleties in

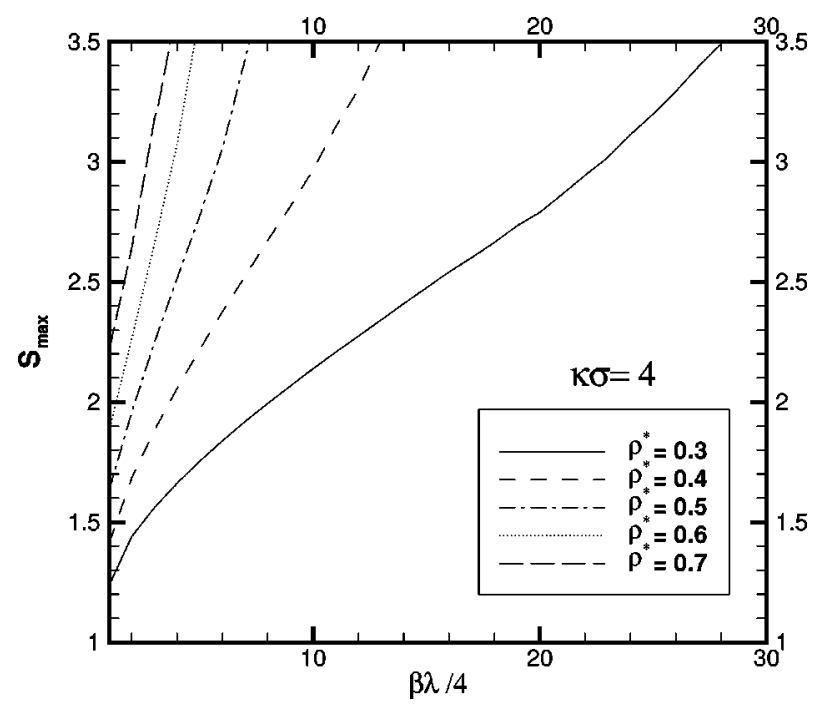

FIG. 7. The value of the first peak in the static structure factor in terms of $\beta \lambda$ at $\kappa \sigma=4$ and $\rho=0.4,0.5,0.6$, and 0.7 .

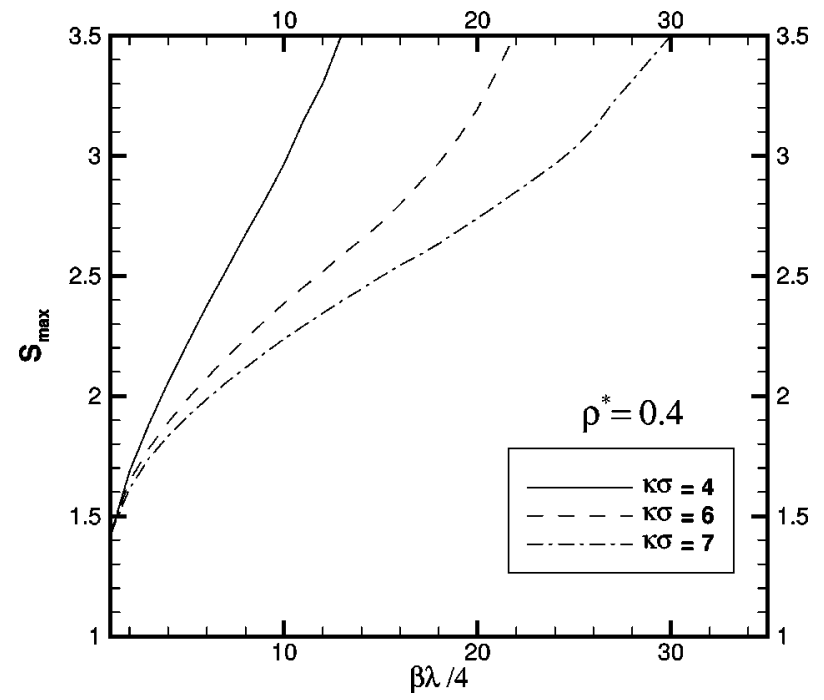

FIG. 8. The value of the first peak in the static structure factor in terms of $\beta \lambda$ at $\rho=0.4$ and $\kappa \sigma=4,6$, and 7 .

freezing or melting transitions. Bladon and Frankel [10], using an attractive square-well potential, found a first order melting transition where the system goes into a liquid phase from a crystal phase. However, molecular dynamics [24] simulations and recent experiments [25] showed solid-tohexatic and hexatic-to-liquid phase transitions if there was an attractive part to the colloidal potential. In an earlier work Lowen [26] did not find a hexatic phase in a Yukawa system. It appears that the way in which the form of the interaction potential influences the possible phases in 2D colloidal systems is not entirely clear. Issues such as attractive or repulsive natures, hard or soft cores, and the long range part of the potential remain to be systematically investigated. In our analysis, we used the Hansen-Verlet criterion to determine the liquid-solid freezing transition, which seems consistent with available results.

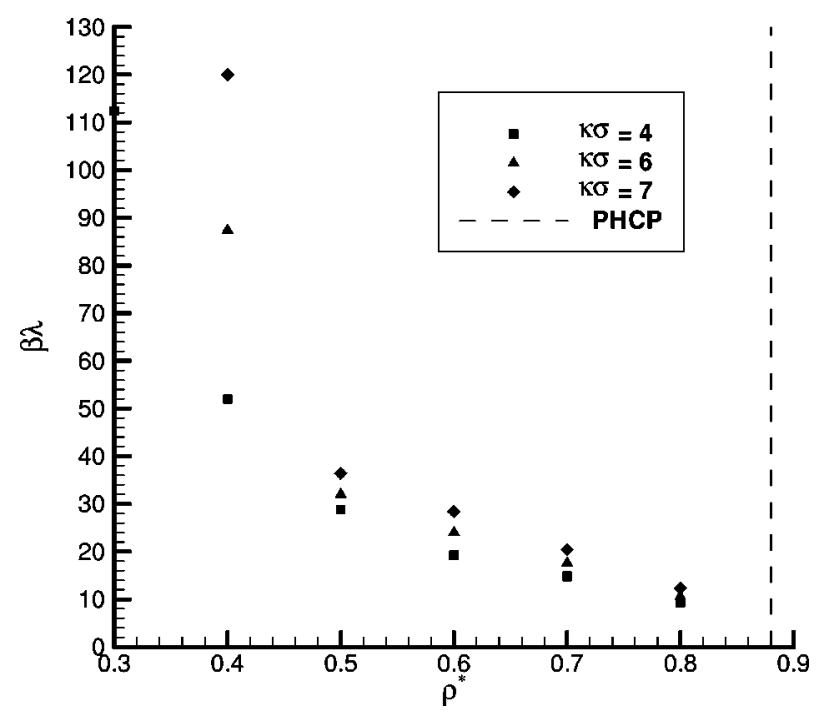

FIG. 9. The liquid-solid phase line transition at $\kappa \sigma=4,6$, and 7 . The dashed line represents the liquid-solid phase transition line for the PHCP. 
In summary, in this paper we have used HNC theory to study correlation functions of a 2D hard-core Yukawa fluid as a model for colloidal systems. We have investigated the effect of the Yukawa tail in the model potential by comparing our results with those for a purely hard-core potential. The reliability of our correlation functions at small distances were tested against the PY approximation. Using the Hansen-Verlet criterion, which uses the value of the first peak in the static structure factor, we obtained a phase diagram showing the liquid-solid transition.

\section{ACKNOWLEDGMENTS}

This work was partially supported by the Scientific and Technical Research Council of Turkey (TUBITAK) under Grant No. TBAG-2005, by NATO under Grant No. SfP971970, and by the Turkish Department of Defense under Grant No. KOBRA-001. One of us (B.D.) acknowledges a grant by Scuola Normale Superiore under the MURST PRIN1999 Initiative. We would like to thank Professor M. P. Tosi and Dr. A. L. Demirel for useful discussions and comments.
[1] A.P. Gast and W.B. Russel, Phys. Today 51(12), 24 (1998).

[2] P. Pieranski, Contemp. Phys. 24, 25 (1983).

[3] Physics of Complex and Supermolecular Fluids, edited by S. A. Safran and N. A. Clark (Wiley, New York, 1987).

[4] P. Pieranski, Phys. Rev. Lett. 45, 569 (1980).

[5] Y. Tang, A.J. Armstrong, R.C. Mockler, and W.J. O'Sullivan, Phys. Rev. Lett. 62, 2401 (1989).

[6] C.A. Murray and D.H. Van Winkle, Phys. Rev. Lett. 58, 1200 (1989).

[7] A.H. Marcus and S.A. Rice, Phys. Rev. Lett. 77, 2577 (1997).

[8] A.H. Marcus and S.A. Rice, Phys. Rev. E 55, 637 (1997).

[9] H. Weber, D. Marx, and K. Binder, Phys. Rev. B 51, 14636 (1995).

[10] P. Bladon and D. Frenkel, Phys. Rev. Lett. 74, 2519 (1995).

[11] T. Terao and T. Nakayama, Phys. Rev. E 60, 7157 (1999).

[12] G.F. Wang and S.K. Lai, Phys. Rev. Lett. 82, 3645 (1999).

[13] Q.-H. Wei, C. Bechinger, D. Rudhardt, and P. Leiderer, Phys. Rev. Lett. 81, 2606 (1998).

[14] R. Bubeck, C. Bechinger, S. Neser, and P. Leiderer, Phys. Rev. Lett. 82, 3364 (1999).

[15] Z.D. Cheng, W.B. Russell, and P.M. Chaikin, Nature (London)
401, 893 (1999); F.M. van der Kooij, K. Kassapidou, and H.N.W. Lekkerkerker, ibid. 406, 868 (2000); S. John and K. Busch, J. Lightwave Technol. 17, 1931 (1999).

[16] Y. Rosenfeld and N.W. Ashcroft, Phys. Rev. A 20, 1208 (1979).

[17] F.J. Rogers and D.A. Young, Phys. Rev. A 30, 999 (1984).

[18] See for a recent application, W. Rzysko, O. Pizio, and S. Sokolowski, Physica A 273, 241 (1999).

[19] B. Davoudi, M. Kohandel, M. Mohammadi, and B. Tanatar, Phys. Rev. E 62, 6977 (2000).

[20] M.G. Noro, M. Kern, and D. Frenkel, Europhys. Lett. 48, 332 (1999).

[21] J.P. Hansen and D.R. Mcdonald, Theory of Simple Liquids, 2nd ed. (Academic, London, 1986).

[22] G.I. Menon, C. Dasgupta, H.R. Krishnamurthy, T.V. Ramakrishnan, and S. Sengupta, Phys. Rev. B 54, 16192 (1996).

[23] J.P. Hansen and L. Verlet, Phys. Rev. 184, 151 (1969).

[24] R. Zangi and S.A. Rice, Phys. Rev. E 58, 7529 (1998).

[25] P. Karnchanaphanurach, B. Lin, and S.A. Rice, Phys. Rev. E 61, 4036 (2000).

[26] H. Löwen, J. Phys.: Condens. Matter 4, 10105 (1992). 